\title{
Analysis of Some Localized Boundary-Domain Integral Equations for Transmission Problems with Variable Coefficients
}

\author{
O. Chkadua ${ }^{1}$, S.E. Mikhailov ${ }^{2}$, and D. Natroshvili ${ }^{3}$ \\ ${ }^{1}$ A.Razmadze Mathematical Institute, Tbilisi, Georgia; chkadua@rmi.acnet.ge \\ ${ }^{2}$ Brunel University, West London, UK; sergey.mikhailov@brunel.ac.uk \\ ${ }^{3}$ Georgian Technical University, Tbilisi, Georgia; natrosh@hotmail.com
}

\subsection{Introduction}

We consider the basic and mixed transmission problems for scalar second order elliptic partial differential equations with variable coefficients and using the localized parametrices reduce the problems to direct segregated boundarydomain integral equations.

The transmission problems treated in the paper are well investigated in the research literature by the variational methods, and the corresponding uniqueness and existence results are well known (see, e.g., [HW08], [LiMa72]).

For the special cases, when the fundamental solution is available, the Dirichlet and Neumann type boundary value problems were also investigated by the classical potential method (see [Mir70], [HW08] and the references therein).

Our goal here is to show that the problems can be equivalently reduced to some localized boundary-domain integral equations (LBDIEs) and the corresponding localized boundary-domain integral operators (LBDIOs) are invertible, which beside a pure mathematical interest may have also some applications in numerical analysis for construction of efficient numerical algorithms (see, e.g., [Mik02], [MN05], [SSA00], [ZZA98], [ZZA99] and the references therein).

In our case, the localized parametrix is represented as the product of a Levi function of the differential operator under consideration and an appropriately chosen localizing function, e.g., a function supported on some neighbourhood of singularity point of the Levi function. Although the kernels of the corresponding localized potentials do not solve the original PDEs, the localized potentials preserve almost all mapping properties of the usual non-localized ones 
(cf. [CMN09-1, Mik06, CMN11, CMN09-L]). However, some unusual properties of the localized potentials appear due to the localization of the kernel functions which have no counterparts in classical potential theory and which need special consideration and analysis.

By the direct approach based on Green's representation formula, we reduce the transmission Dirichlet and mixed type problems to the LBDIE system. First we establish the equivalence between the original transmission problems and the corresponding LBDIE systems, which proved to be a quite nontrivial problem and plays a crucial role in our analysis. Afterwards we investigate Fredholm properties of the LBDIOs and prove their invertibility in appropriate function spaces. In this paper we present analysis for a wider classes of the localizing functions than in [CMN09-L].

\subsection{Reduction to localized boundary-domain integral equations}

\subsubsection{Formulation of the interface problems}

Let $\Omega$ and $\Omega_{1}$ be bounded open domains in $\mathbb{R}^{3}, \bar{\Omega}_{1} \subset \Omega$ and $\Omega_{2}:=\Omega \backslash \bar{\Omega}_{1}$. We assume that the interface surface $S_{i}=\partial \Omega_{1}$ and the exterior boundary $S_{e}=\partial \Omega$ of the composite body $\bar{\Omega}=\bar{\Omega}_{1} \cup \bar{\Omega}_{2}$ are sufficiently smooth, say $C^{\infty}$-regular if not otherwise stated. Clearly, $\partial \Omega_{2}=S_{i} \cup S_{e}$. Throughout the paper $n^{(q)}=n^{(q)}(x)$ denotes the unit normal vector to $\partial \Omega_{q}$ directed outward the corresponding domain $\Omega_{q}$. Clearly, $n^{(1)}(x)=-n^{(2)}(x)$ for $x \in S_{i}$.

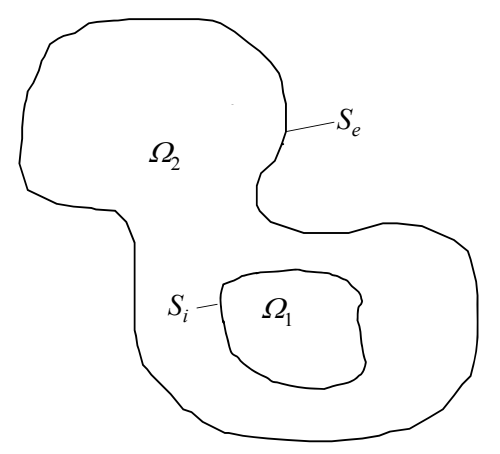

By $H^{r}\left(\Omega^{\prime}\right)=H_{2}^{r}\left(\Omega^{\prime}\right)$ and $H^{r}(S)=H_{2}^{r}(S), r \in \mathbb{R}$, we denote the Bessel potential spaces on a domain $\Omega^{\prime}$ and on a closed manifold $S$ without boundary. The subspace of $H^{r}\left(\mathbb{R}^{3}\right)$ of functions with compact support is denoted by $H_{\text {comp }}^{r}\left(\mathbb{R}^{3}\right)$. Recall that $H^{0}\left(\Omega^{\prime}\right)=L_{2}\left(\Omega^{\prime}\right)$ is a space of square integrable functions in $\Omega$.

For a smooth sub-manifold $\mathcal{M} \subset S$ we denote by $\widetilde{H}^{r}(\mathcal{M})$ the subspace of $H^{r}(S), \widetilde{H}^{r}(\mathcal{M}):=\left\{g: g \in H^{r}(\mathcal{M}), \operatorname{supp} g \subset \overline{\mathcal{M}}\right\}$, while $H^{r}(\mathcal{M})$ denotes the 
spaces of restrictions on $\mathcal{M}$ of functions from $H^{r}(S), H^{r}(\mathcal{M}):=\left\{r_{\mathcal{M}} f: f \in\right.$ $\left.H^{r}(S)\right\}$, where $r_{\mathcal{M}}$ is the restriction operator onto $\mathcal{M}$.

Let us consider the differential operators in the domains $\Omega_{q}$

$$
A_{q}\left(x, \partial_{x}\right) u(x):=\sum_{k=1}^{3} \partial_{x_{k}}\left[a_{q}(x) \partial_{x_{k}} u(x)\right], \quad q=1,2,
$$

where $\partial_{x}=\left(\partial_{1}, \partial_{2}, \partial_{3}\right), \partial_{k}=\partial_{x_{k}}=\partial / \partial x_{k}, k=1,2,3$, and

$$
a_{q} \in C^{\infty}\left(\mathbb{R}^{3}\right), \quad 0<c_{0} \leq a_{q}(x) \leq c_{1}<\infty, \quad q=1,2 .
$$

Further, for sufficiently smooth functions (from the space $H^{2}\left(\Omega_{q}\right)$, say) we introduce the co-normal derivative operator on $\partial \Omega_{q}, q=1,2$, in the usual trace sense:

$$
T_{q}^{ \pm}\left(x, \partial_{x}\right) u(x):=\sum_{k=1}^{3} a_{q}(x) n_{k}^{(q)}(x) \gamma_{q}^{ \pm}\left[\partial_{x_{k}} u(x)\right],
$$

where $x \in \partial \Omega_{q}$ and the symbol $\gamma_{q}^{+}$and $\gamma_{q}^{-}$denote the trace operators on $\partial \Omega_{q}$ from the domain $\Omega_{q}^{+}:=\Omega_{q}$ and its complement $\Omega_{q}^{-}:=\mathbb{R}^{3} \backslash \bar{\Omega}_{q}$, respectively.

We set

$$
H^{1,0}\left(\Omega_{q}^{ \pm} ; A_{q}\right):=\left\{u \in H^{1}\left(\Omega_{q}^{ \pm}\right): A_{q} u \in H^{0}\left(\Omega_{q}^{ \pm}\right)\right\}, \quad q=1,2 .
$$

The classical co-normal derivative operators given by (1.1) can be continuously extended to functions from the spaces $H^{1,0}\left(\Omega_{q}^{ \pm} ; A_{q}\right)$ by the (generalized) canonical co-normal derivative operators $T_{q}^{ \pm}: H^{1,0}\left(\Omega_{q}^{ \pm} ; A_{q}\right) \rightarrow H^{-\frac{1}{2}}\left(\partial \Omega_{q}\right)$ (cf., for example, [Co88, Lemma 3.2], [McL00, Lemma 4.3]) defined as

$$
\left\langle T_{q}^{ \pm} u, w\right\rangle_{\partial \Omega_{q}}:= \pm \int_{\Omega_{q}}\left[\left(\ell_{q}^{ \pm} w\right) A_{q} u+E_{q}\left(u, \ell_{q}^{ \pm} w\right)\right] d x
$$

for all $w \in H^{\frac{1}{2}}\left(\partial \Omega_{q}\right)$. Here $\ell_{q}^{ \pm}$are continuous linear extension operators, $\ell_{q}^{ \pm}: H^{\frac{1}{2}}\left(\partial \Omega_{q}\right) \rightarrow H^{1}\left(\Omega_{q}^{ \pm}\right)$which are right inverse to the trace operators $\gamma_{q}^{ \pm}$, while

$$
E_{q}(u, v):=a_{q}(x) \nabla_{x} u \cdot \nabla_{x} v, \quad \nabla_{x}:=\left(\partial_{1}, \partial_{2}, \partial_{3}\right)^{\top},
$$

and the central dot denotes the scalar product in $\mathbb{R}^{3}$. The symbol $\left\langle g_{1}, g_{2}\right\rangle_{\partial \Omega_{q}}$ in (1.2) denotes the duality brackets between the spaces $H^{-\frac{1}{2}}\left(\partial \Omega_{q}\right)$ and $H^{\frac{1}{2}}\left(\partial \Omega_{q}\right)$, coinciding with $\int_{\partial \Omega_{q}} g_{1}(x) g_{2}(x) d S$ if $g_{1}, g_{2} \in L_{2}\left(\partial \Omega_{q}\right)$. Below for these type dualities we will use sometimes the usual integral symbol when this does not lead to confusion. We will also employ the shorter notations $\gamma_{q} \equiv \gamma_{q}^{+}$, $T_{q} \equiv T_{q}^{+}$.

Now we formulate the following Dirichlet and mixed type transmission problems: 
Find functions $u_{1} \in H^{1,0}\left(\Omega_{1} ; A_{1}\right)$ and $u_{2} \in H^{1,0}\left(\Omega_{2} ; A_{2}\right)$ satisfying the differential equations

$$
A_{q}\left(x, \partial_{x}\right) u_{q}=f_{q} \quad \text { in } \Omega_{q}, \quad q=1,2,
$$

the transmission conditions on the interface

$$
\begin{array}{ll}
\gamma_{1} u_{1}-\gamma_{2} u_{2}=\varphi_{0 i} & \text { on } \quad S_{i}, \\
T_{1} u_{1}+T_{2} u_{2}=\psi_{0 i} & \text { on } \quad S_{i},
\end{array}
$$

and one of the following conditions on the exterior boundary: the Dirichlet boundary condition

$$
\gamma_{2} u_{2}=\varphi_{0 e} \quad \text { on } S_{e},
$$

or the mixed type boundary conditions

$$
\begin{array}{llll}
\gamma_{2} u_{2}=\varphi_{0 e}^{(M)} & \text { on } & S_{e D}, \\
T_{2} u_{2}=\psi_{0 e}^{(M)} & \text { on } & S_{e N},
\end{array}
$$

where $S_{e D}$ and $S_{e N}$ are smooth disjoint sub-manifolds of $S_{e}: S_{e}=\bar{S}_{e D} \cup \bar{S}_{e N}$ and $S_{e D} \cap S_{e N}=\varnothing$.

We will refer to these boundary transmission problems as (TD) and (TM) problems, respectively.

For the data in the above formulated problems we assume

$$
\begin{gathered}
\varphi_{0 i} \in H^{\frac{1}{2}}\left(S_{i}\right), \quad \psi_{0 i} \in H^{-\frac{1}{2}}\left(S_{i}\right), \quad \varphi_{0 e} \in H^{\frac{1}{2}}\left(S_{e}\right), \quad \psi_{0 e} \in H^{-\frac{1}{2}}\left(S_{e}\right), \\
\varphi_{0 e}^{(M)} \in H^{\frac{1}{2}}\left(S_{e D}\right), \quad \psi_{0 e}^{(M)} \in H^{-\frac{1}{2}}\left(S_{e N}\right), \quad f_{q} \in H^{0}\left(\Omega_{q}\right), \quad q=1,2 .
\end{gathered}
$$

The equations (1.3) are understood in the distributional sense, the Dirichlet type boundary and transmission conditions are understood in the usual trace sense, while the Neumann type conditions for the co-normal derivatives are understood in the sense of the canonical co-normal derivatives defined by (1.2).

We recall that the normal vectors $n^{(1)}$ and $n^{(2)}$ in the definitions of the co-normal derivatives $T_{1} u$ and $T_{2} u$ on $S_{i}$ have opposite directions.

As we have mentioned in the introduction, all the above formulated transmission problems are well investigated in the literature by the variational methods and the corresponding uniqueness and existence results are well known (see, e.g., [LiMa72]). Our goal here is to show that the problems can be equivalently reduced to some LBDIEs and to investigate the Fredholm and invertibility properties of the corresponding LBDIOs.

\subsubsection{Properties of localized potentials}

It is well known that the function 


$$
P_{q 1}(x, y)=-\frac{1}{4 \pi a_{q}(y)|x-y|}
$$

is a Levi function for the operator $A_{q}\left(x, \partial_{x}\right)$ (cf. [CMN09-1]).

Now we introduce the localized parametrix (localized Levi function) for the operator $A_{q}$,

$$
P_{q}(x, y) \equiv P_{q \chi}(x, y):=\chi(x-y) P_{q 1}(x, y), \quad q=1,2,
$$

where $\chi$ is a localizing function (see Appendix A)

$$
\chi(x)=\breve{\chi}(|x|), \quad \chi \in X_{1 *}^{k}, \quad k \geq 3 .
$$

One can easily check the following relation [CMN09-L],

$$
A_{q}\left(x, \partial_{x}\right) P_{q}(x, y)=\delta(x-y)+R_{q}(x, y), \quad q=1,2,
$$

where $\delta(\cdot)$ is the Dirac distribution and

$$
\begin{gathered}
R_{q}(x, y)=R_{q \chi}(x, y)=-\frac{1}{4 \pi a_{q}(y)} \sum_{j=1}^{3}\left\{-\frac{\partial}{\partial y_{j}}\left[\frac{\partial a_{q}(x)}{\partial x_{j}} \frac{\chi(x-y)}{|x-y|}\right.\right. \\
\left.\left.+a_{q}(x) \frac{\partial \chi(x-y)}{\partial x_{j}} \frac{1}{|x-y|}\right]+a_{q}(x) \frac{\partial \chi(x-y)}{\partial x_{j}} \frac{\partial}{\partial x_{j}} \frac{1}{|x-y|}\right\} .
\end{gathered}
$$

The function $R_{q}(x, y)$ possesses a weak singularity of type $\mathcal{O}\left(|x-y|^{-2}\right)$ as $x \rightarrow y$ if $\chi$ is smooth enough, e.g., if $\chi \in X^{2}$.

Let us introduce the localized volume potentials for $y \in \mathbb{R}^{3}$,

$$
\begin{aligned}
& \mathcal{P}_{q} f(y):=\int_{\Omega_{q}} P_{q}(x, y) f(x) d x, \\
& \mathcal{R}_{q} f(y):=\int_{\Omega_{q}} R_{q}(x, y) f(x) d x,
\end{aligned}
$$

and the surface potentials for $y \in \mathbb{R}^{3} \backslash S$,

$$
\begin{aligned}
& V_{S}^{(q)} g(y):=-\int_{S} P_{q}(x, y) g(x) d S_{x}, \\
& W_{S}^{(q)} g(y):=-\int_{S}\left[T_{q}\left(x, \partial_{x}\right) P_{q}(x, y)\right] g(x) d S_{x},
\end{aligned}
$$

based on the localized parametrices $P_{q}$. Here $S \in\left\{S_{i}, S_{e}, \partial \Omega_{2}\right\}$. Note that for the layer potentials we will drop the subindex $S$ when $S=\partial \Omega_{q}$, i.e., $V^{(q)}:=V_{\partial \Omega_{q}}^{(q)}, W^{(q)}:=W_{\partial \Omega_{q}}^{(q)}$. If the domain of integration in (1.9) is replaced with the whole space $\Omega_{q}=\mathbb{R}^{3}$, we employ the notation $\mathbf{P}_{q} f=\mathcal{P}_{q} f$.

Let us also define the corresponding boundary operators generated by the direct values of the localized single and double layer potentials and their conormal derivatives for $y \in S$ 


$$
\begin{aligned}
& \mathcal{V}_{S}^{(q)} g(y):=-\int_{S} P_{q}(x, y) g(x) d S_{x}, \\
& \mathcal{W}_{S}^{(q)} g(y):=-\int_{S}\left[T_{q}\left(x, \partial_{x}\right) P_{q}(x, y)\right] g(x) d S_{x}, \\
& \mathcal{W}_{S}^{(q)} g(y):=-\int_{S}\left[T_{q}\left(y, \partial_{y}\right) P_{q}(x, y)\right] g(x) d S_{x}, \\
& \mathcal{L}_{S}^{ \pm(q)} g(y):=r_{S} T_{q}^{ \pm}\left(y, \partial_{y}\right) W_{S}^{(q)} g(y) .
\end{aligned}
$$

For the pseudodifferential operator (1.13), we employ also the notation $\mathcal{L}_{S}^{(q)}:=$ $\mathcal{L}_{S}^{+(q)}$. Note that the kernel functions of the operators (1.11) and (1.12) are at most weakly singular if the localizing function $\chi \in X^{2}$ and the surface $S$ is $C^{1, \beta}$ smooth with $\beta>0$. Mapping properties of the operators (1.9)-(1.13) are studied in [CMN09-L].

Further on we assume that the following relation holds on the interface,

$$
a_{2}(x)=\varkappa a_{1}(x) \text { for } x \in S_{i}, \quad \varkappa=\text { const }>0 .
$$

Finally, we present some auxiliary propositions which play a crucial role in our analysis and which can be proved by extending the arguments similar to those applied in the proof of Lemmas 6.3 and 6.4 in [CMN09-L] from the case $\chi \in X_{1+}^{3}$ to the case $\chi \in X_{1 *}^{3}$.

Lemma 1. Let $\chi \in X_{1 *}^{3}$ and condition (1.14) hold. Further let $G_{q} \in H^{0}\left(\Omega_{q}\right)$, $g_{1} \in H^{-\frac{1}{2}}\left(S_{i}\right), g_{2} \in H^{\frac{1}{2}}\left(S_{i}\right), g_{e} \in H^{-\frac{1}{2}}\left(S_{e}\right)$ and

$$
\begin{aligned}
& V_{S_{i}}^{(1)}\left(g_{1}\right)+W_{S_{i}}^{(1)}\left(g_{2}\right)+\mathcal{P}_{1}\left(G_{1}\right)=0 \text { in } \Omega_{1}, \\
& V_{S_{i}}^{(2)}\left(g_{1}\right)-W_{S_{i}}^{(2)}\left(g_{2}\right)+V_{S_{e}}^{(2)}\left(g_{e}\right)+\mathcal{P}_{2}\left(G_{2}\right)=0 \text { in } \Omega_{2} .
\end{aligned}
$$

Then $G_{q}=0$ in $\Omega_{q}, q=1,2, g_{1}=0, g_{2}=0$ on $S_{i}$, and $g_{e}=0$ on $S_{e}$.

Lemma 2. Let $\chi \in X_{1 *}^{3}$ and condition (1.14) hold. Further let $G_{q} \in H^{0}\left(\Omega_{q}\right)$, $g_{1} \in H^{-\frac{1}{2}}\left(S_{i}\right), g_{2} \in H^{\frac{1}{2}}\left(S_{i}\right), g_{e D} \in \widetilde{H}^{-\frac{1}{2}}\left(S_{e D}\right), g_{e N} \in \widetilde{H}^{\frac{1}{2}}\left(S_{e N}\right)$, and

$$
\begin{aligned}
& V_{S_{i}}^{(1)}\left(g_{1}\right)+W_{S_{i}}^{(1)}\left(g_{2}\right)+\mathcal{P}_{1}\left(G_{1}\right)=0 \text { in } \Omega_{1}, \\
& V_{S_{i}}^{(2)}\left(g_{1}\right)-W_{S_{i}}^{(2)}\left(g_{2}\right)+V_{S_{e}}^{(2)}\left(g_{e D}\right)+W_{S_{e}}^{(2)}\left(g_{e N}\right)+\mathcal{P}_{2}\left(G_{2}\right)=0 \text { in } \Omega_{2} .
\end{aligned}
$$

Then $G_{q}=0$ in $\Omega_{q}, q=1,2, g_{1}=0$ and $g_{2}=0$ on $S_{i}, g_{e D}=0$ and $g_{e N}=0$ on $S_{e}$.

\subsubsection{Basic LBDIE relations}

Second Green's identity holds for the operator $A_{q}\left(x, \partial_{x}\right)$ and $u, v \in H^{1,0}\left(\Omega_{q} ; A_{q}\right)$, see, e.g., [Co88, Lemma 3.2], [McL00, Lemma 4.3],

$$
\int_{\Omega_{q}}\left[v A_{q} u-u A_{q} v\right] d x=\int_{\partial \Omega_{q}}\left[\left(\gamma_{q} v\right) T_{q} u-\left(\gamma_{q} u\right) T_{q} v\right] d S, \quad q=1,2 .
$$


By the standard limiting procedure near the singular point of the parametrix (see, e.g., [Mir70]), we obtain the following parametrix-based third Green's identity for arbitrary $u=u_{q} \in H^{1,0}\left(\Omega_{q} ; A_{q}\right)$,

$$
u_{q}+\mathcal{R}_{q} u_{q}-V^{(q)} T_{q} u_{q}+W^{(q)} \gamma_{q} u_{q}=\mathcal{P}_{q} A_{q} u_{q} \text { in } \Omega_{q} .
$$

Recall that for layer potentials we drop the subindex $S$ when $S=\partial \Omega_{q}$.

Taking in mind the properties of the localized potentials exposed in Appendix $\mathrm{B}$, for the trace and co-normal derivative of (1.15) we get

$$
\begin{aligned}
& \frac{1}{2} \gamma_{q} u_{q}+\gamma_{q} \mathcal{R}_{q} u_{q}-\mathcal{V}^{(q)} T_{q} u_{q}+\mathcal{W}^{(q)} \gamma_{q} u_{q}=\gamma_{q} \mathcal{P}_{q} A_{q} u_{q} \text { on } \partial \Omega_{q} \\
& \frac{1}{2} T_{q} u_{q}+T_{q} \mathcal{R}_{q} u_{q}-\mathcal{W}^{\prime(q)} T_{q} u_{q}+\mathcal{L}^{(q)} \gamma_{q} u_{q}=T_{q} \mathcal{P}_{q} A_{q} u_{q} \text { on } \partial \Omega_{q}
\end{aligned}
$$

With the help of these relations one can construct various types of LBDIE systems for the above formulated transmission BVPs.

\subsection{LBDIES for the transmission Dirichlet problem}

Let a pair $\left(u_{1}, u_{2}\right) \in H^{1,0}\left(\Omega_{1} ; A_{1}\right) \times H^{1,0}\left(\Omega_{2} ; A_{2}\right)$ be a solution to the transmission Dirichlet problem (1.3)-(1.6), i.e., Problem (TD). Assume that the problem right hand sides satisfy the imbeddings

$$
\varphi_{0 i} \in H^{\frac{1}{2}}\left(S_{i}\right), \psi_{0 i} \in H^{-\frac{1}{2}}\left(S_{i}\right), \varphi_{0 e} \in H^{\frac{1}{2}}\left(S_{e}\right), f_{q} \in H^{0}\left(\Omega_{q}\right) .
$$

Let us introduce the following combinations of the unknown functions

$$
\psi_{i}=\frac{1}{2} r_{S_{i}}\left(T_{1} u_{1}-T_{2} u_{2}\right), \quad \varphi_{i}=\frac{1}{2} r_{S_{i}}\left(\gamma_{1} u_{1}+\gamma_{2} u_{2}\right), \quad \psi_{e}=r_{S_{e}} T_{2} u_{2} .
$$

Then evidently $\psi_{i} \in H^{-\frac{1}{2}}\left(S_{i}\right), \varphi_{i} \in H^{\frac{1}{2}}\left(S_{i}\right), \psi_{e} \in H^{-\frac{1}{2}}\left(S_{e}\right)$.

Let us introduce the five-vector function (column matrix function)

$$
U^{(T D)}:=\left(u_{1}, u_{2}, \psi_{i}, \varphi_{i}, \psi_{e}\right)^{\top} \in \mathbb{H}^{(T D)},
$$

where

$$
\mathbb{H}^{(T D)}:=H^{1,0}\left(\Omega_{1} ; A_{1}\right) \times H^{1,0}\left(\Omega_{2} ; A_{2}\right) \times H^{-\frac{1}{2}}\left(S_{i}\right) \times H^{\frac{1}{2}}\left(S_{i}\right) \times H^{-\frac{1}{2}}\left(S_{e}\right),
$$

and consider formally the components of $U^{(T D)}$ as unrelated to each other (i.e., segregated).

Further, let us employ the third Green identities (1.15) in $\Omega_{1}$ and $\Omega_{2}$, difference of their traces (1.16) and sum of their co-normal derivatives (1.17) on $S_{i}$, and also the trace (1.16) on $S_{e}$. Then after substituting transmission and boundary conditions (1.4)-(1.6) and notations (1.19) we arrive at the following 
system of direct segregated LBDIEs for the components of the vector function $U^{(T D)}=\left(u_{1}, u_{2}, \psi_{i}, \varphi_{i}, \psi_{e}\right)^{\top}$,

$$
\begin{gathered}
u_{1}+\mathcal{R}_{1} u_{1}-V_{S_{i}}^{(1)} \psi_{i}+W_{S_{i}}^{(1)} \varphi_{i}=F_{1}^{(T D)} \text { in } \Omega_{1}, \\
u_{2}+\mathcal{R}_{2} u_{2}+V_{S_{i}}^{(2)} \psi_{i}+W_{S_{i}}^{(2)} \varphi_{i}-V_{S_{e}}^{(2)} \psi_{e}=F_{2}^{(T D)} \text { in } \Omega_{2}, \\
\gamma_{1} \mathcal{R}_{1} u_{1}-\gamma_{2} \mathcal{R}_{2} u_{2}-\left(\mathcal{V}_{S_{i}}^{(1)}+\mathcal{V}_{S_{i}}^{(2)}\right) \psi_{i}+\left(\mathcal{W}_{S_{i}}^{(1)}-\mathcal{W}_{S_{i}}^{(2)}\right) \varphi_{i}+\gamma_{2} V_{S_{e}}^{(2)} \psi_{e} \\
=\gamma_{1} F_{1}^{(T D)}-\gamma_{2} F_{2}^{(T D)}-\varphi_{0 i} \text { on } S_{i}, \\
T_{1} \mathcal{R}_{1} u_{1}+T_{2} \mathcal{R}_{2} u_{2}-\left(\mathcal{W}_{S_{i}}^{(1)}-\mathcal{W}_{S_{i}}^{(2)}\right) \psi_{i}+\left(\mathcal{L}_{S_{i}}^{(1)}+\mathcal{L}_{S_{i}}^{(2)}\right) \varphi_{i}-T_{2} V_{S_{e}}^{(2)} \psi_{e} \\
=T_{1} F_{1}^{(T D)}+T_{2} F_{2}^{(T D)}-\psi_{0 i} \text { on } S_{i}, \\
\gamma_{2} \mathcal{R}_{2} u_{2}+\gamma_{2} V_{S_{i}}^{(2)} \psi_{i}+\gamma_{2} W_{S_{i}}^{(2)} \varphi_{i}-\mathcal{V}_{S_{e}}^{(2)} \psi_{e}=\gamma_{2} F_{2}^{(T D)}-\varphi_{0 e} \text { on } S_{e},
\end{gathered}
$$

where

$$
\begin{aligned}
F_{1}^{(T D)} & =\mathcal{P}_{1} f_{1}+\frac{1}{2} V_{S_{i}}^{(1)} \psi_{0 i}-\frac{1}{2} W_{S_{i}}^{(1)} \varphi_{0 i} \\
F_{2}^{(T D)} & =\mathcal{P}_{2} f_{2}+\frac{1}{2} V_{S_{i}}^{(2)} \psi_{0 i}+\frac{1}{2} W_{S_{i}}^{(2)} \varphi_{0 i}-W_{S_{e}}^{(2)} \varphi_{0 e}
\end{aligned}
$$

If we introduce the notation

$$
\begin{gathered}
\mathcal{K}^{(T D)}=\left[\mathcal{K}_{k j}^{(T D)}\right]_{5 \times 5}:= \\
{\left[\begin{array}{ccccc}
I+r_{\Omega_{1}} \mathcal{R}_{1} & 0 & -r_{\Omega_{1}} V_{S_{i}}^{(1)} & r_{\Omega_{1}} W_{S_{i}}^{(1)} & 0 \\
0 & I+r_{\Omega_{2}} \mathcal{R}_{2} & r_{\Omega_{2}} V_{S_{i}}^{(2)} & r_{\Omega_{2}} W_{S_{i}}^{(2)} & -r_{\Omega_{2}} V_{S_{e}}^{(2)} \\
r_{S_{i}} \gamma_{1} \mathcal{R}_{1} & -r_{S_{i}} \gamma_{2} \mathcal{R}_{2} & -\mathcal{V}_{S_{i}}^{(1)}-\mathcal{V}_{S_{i}}^{(2)} & \mathcal{W}_{S_{i}}^{(1)}-\mathcal{W}_{S_{i}}^{(2)} & r_{S_{i}} \gamma_{2} V_{S_{e}}^{(2)} \\
r_{S_{i}} T_{1} \mathcal{R}_{1} & r_{S_{i}} T_{2} \mathcal{R}_{2} & -\mathcal{W}_{S_{i}}^{(1)}+\mathcal{W}_{S_{i}}^{(2)} & \mathcal{L}_{S_{i}}^{(1)}+\mathcal{L}_{S_{i}}^{(2)} & -r_{S_{i}} T_{2} V_{S_{e}}^{(2)} \\
0 & r_{S_{e}} \gamma_{2} \mathcal{R}_{2} & r_{S_{e}} \gamma_{2} V_{S_{i}}^{(2)} & r_{S_{e}} \gamma_{2} W_{S_{i}}^{(2)} & -\mathcal{V}_{S_{e}}^{(2)}
\end{array}\right],}
\end{gathered}
$$

the LBDIEs system (1.22)-(1.26) can be rewritten as

$$
\mathcal{K}^{(T D)} U^{(T D)}=\mathcal{F}^{(T D)},
$$

where $U^{(T D)} \in \mathbb{H}^{(T D)}$ is the unknown vector, while $\mathcal{F}^{(T D)} \in \mathbb{F}^{(T D)}$ is the known vector generated by the right hand side functions in (1.22)-(1.26) and

$$
\mathbb{F}^{(T D)}:=H^{1,0}\left(\Omega_{1} ; A_{1}\right) \times H^{1,0}\left(\Omega_{2} ; A_{2}\right) \times H^{\frac{1}{2}}\left(S_{i}\right) \times H^{-\frac{1}{2}}\left(S_{i}\right) \times H^{\frac{1}{2}}\left(S_{e}\right) .
$$

There holds the following equivalence theorem.

Theorem 1. Let conditions (1.18) hold and $\chi \in X_{1 *}^{3}$.

(i) If a pair $\left(u_{1}, u_{2}\right) \in H^{1,0}\left(\Omega_{1} ; A_{1}\right) \times H^{1,0}\left(\Omega_{2} ; A_{2}\right)$ solves the Problem (TD), then the five-vector $U^{(T D)} \in \mathbb{H}^{(T D)}$ given by $(1.20)$, where $\psi_{i}, \varphi_{i}$ and $\psi_{e}$ are defined by (1.19), solves LBDIEs system (1.22)-(1.26). 
(ii) Vice versa, if a five-vector $U^{(T D)} \in \mathbb{H}^{(T D)}$ solves LBDIEs system (1.22)-(1.26) and condition (1.14) holds, then $\left(u_{1}, u_{2}\right) \in H^{1,0}\left(\Omega_{1} ; A_{1}\right) \times$ $H^{1,0}\left(\Omega_{2} ; A_{2}\right)$ solves Problem (TD) and relations (1.19) hold.

Proof. Claim (i) immediately follows from the deduction of (1.22)-(1.26).

Now, let a five-vector $U^{(T D)} \in \mathbb{H}^{(T D)}$ solve LBDIEs system (1.22)-(1.26). Subtracting from equation (1.24) the trace $\gamma_{1}$ of equation (1.22) and adding the trace $\gamma_{2}$ of equation (1.23), we prove (1.4). Similarly, subtracting from equation (1.25) the co-normal derivative $T_{1}$ of equation (1.22) and the conormal derivative $T_{2}$ of equation (1.23), we prove (1.5). At last, subtracting from equation (1.26) the trace $\gamma_{2}$ of equation (1.23), we prove (1.6). That is, the transmission conditions on $S_{i}$ and the Dirichlet boundary condition on $S_{e}$ are fulfilled.

It remains to show that $u_{q}$ solves the differential equations (1.3) and that the conditions (1.19) hold true. Due to the embedding $U^{(T D)} \in \mathbb{H}^{(T D)}$, the third Green identities (1.15) hold. Comparing these identities with the first two equations of the LBDIEs system, (1.22) and (1.23), and taking into account transmission conditions (1.4)-(1.5) and the Dirichlet boundary condition (1.6), already satisfied, we arrive at the relations

$$
\begin{aligned}
V_{S_{i}}^{(1)}\left(\frac{1}{2}\left[T_{1} u_{1}-T_{2} u_{2}\right]-\psi_{i}\right)+ & W_{S_{i}}^{(1)}\left(\varphi_{i}-\frac{1}{2}\left[\gamma_{1} u_{1}+\gamma_{2} u_{2}\right]\right) \\
& =\mathcal{P}_{1}\left(f_{1}-A_{1} u_{1}\right) \text { in } \Omega_{1}, \\
V_{S_{i}}^{(2)}\left(\frac{1}{2}\left[T_{1} u_{1}-T_{2} u_{2}\right]-\psi_{i}\right)- & W_{S_{i}}^{(2)}\left(\varphi_{i}-\frac{1}{2}\left[\gamma_{1} u_{1}+\gamma_{2} u_{2}\right]\right) \\
& +V_{S_{e}}^{(2)}\left(\psi_{e}-T_{2} u_{2}\right)=\mathcal{P}_{2}\left(A_{2} u_{2}-f_{2}\right) \text { in } \Omega_{2} .
\end{aligned}
$$

Whence by Lemma 1 we conclude that conditions (1.19) are satisfied and

$$
A_{1} u_{1}-f_{1}=0 \text { in } \Omega_{1}, \quad A_{2} u_{2}-f_{2}=0 \text { in } \Omega_{2} .
$$

This completes the proof.

Due to this equivalence theorem we conclude that the LBDIE system (1.22)-(1.26) with the special right hand side which belongs to the space $\mathbb{F}^{(T D)}$ is uniquely solvable in the space $\mathbb{H}^{(T D)}$ defined by (1.21). In particular, the corresponding homogeneous LBDIEs system possesses only the trivial solution. By the way, one can easily check that the right hand side expressions in LBDIEs system (1.22)-(1.26) vanish if $f_{q}=0$ in $\Omega_{q}, q=1,2, \varphi_{0 i}=0$ and $\psi_{0 i}=0$ on $S_{i}$, and $\varphi_{0 e}=0$ on $S_{e}$.

Our next aim is to establish the invertibility of the matrix operator generated by the left hand side expressions in the LBDIEs system (1.22)-(1.26) both in already introduced and in wider function spaces.

Let us introduce the notations

$$
\begin{aligned}
\mathbb{X}^{(T D)} & :=H^{1}\left(\Omega_{1}\right) \times H^{1}\left(\Omega_{2}\right) \times H^{-\frac{1}{2}}\left(S_{i}\right) \times H^{\frac{1}{2}}\left(S_{i}\right) \times H^{-\frac{1}{2}}\left(S_{e}\right), \\
\mathbb{Y}^{(T D)} & :=H^{1}\left(\Omega_{1}\right) \times H^{1}\left(\Omega_{2}\right) \times H^{\frac{1}{2}}\left(S_{i}\right) \times H^{-\frac{1}{2}}\left(S_{i}\right) \times H^{\frac{1}{2}}\left(S_{e}\right) .
\end{aligned}
$$


Evidently $\mathbb{H}^{(T D)} \subset \mathbb{X}^{(T D)}$ and $\mathbb{F}^{(T D)} \subset \mathbb{Y}^{(T D)}$.

Due to Theorems 6 and 7 in Appendix B the following operators are bounded if $\chi \in X^{3}$,

$$
\begin{aligned}
\mathcal{K}^{(T D)} & : \mathbb{H}^{(T D)} \rightarrow \mathbb{F}^{(T D)} \\
& : \mathbb{X}^{(T D)} \rightarrow \mathbb{Y}^{(T D)}
\end{aligned}
$$

Theorem 2. Let $\chi \in X_{1 *}^{3}$ and condition (1.14) hold. Then the operators (1.28) and (1.29) are invertible.

Proof. We can easily see that the upper triangular matrix operator

$$
\mathcal{K}_{0}^{(T D)}:=\left[\begin{array}{ccccc}
I & 0 & -r_{\Omega_{1}} V_{S_{i}}^{(1)} & r_{\Omega_{1}} W_{S_{i}}^{(1)} & 0 \\
0 & I & r_{\Omega_{2}} V_{S_{i}}^{(2)} & r_{\Omega_{2}} W_{S_{i}}^{(2)} & -r_{\Omega_{2}} V_{S_{e}}^{(2)} \\
0 & 0 & -\mathcal{V}_{S_{i}}^{(1)}-\mathcal{V}_{S_{i}}^{(2)} & \mathcal{W}_{S_{i}}^{(1)}-\mathcal{W}_{S_{i}}^{(2)} & r_{S_{i}} \gamma_{2} V_{S_{e}}^{(2)} \\
0 & 0 & 0 & \mathcal{L}_{S_{i}}^{(1)}+\mathcal{L}_{S_{i}}^{(2)} & -r_{S_{i}} T_{2} V_{S_{e}}^{(2)} \\
0 & 0 & 0 & 0 & -\mathcal{V}_{S_{e}}^{(2)}
\end{array}\right]
$$

possesses the same mapping properties as the operator $\mathcal{K}^{(T D)}$,

$$
\mathcal{K}_{0}^{(T D)}: \mathbb{X}^{(T D)} \rightarrow \mathbb{Y}^{(T D)},
$$

and by Lemma 4 in Appendix B the operator (1.31) is a compact perturbation of the operator (1.29).

For $q=1,2$, the operators $\mathcal{V}_{S}^{(q)}: H^{-\frac{1}{2}}(S) \rightarrow H^{\frac{1}{2}}(S)$ are strongly elliptic pseudodifferential operators of order -1 with strictly positive principal homogenous symbols $\left[2 a_{q}(y)\left|\xi^{\prime}\right|\right]^{-1}$ for $\xi^{\prime} \in \mathbb{R}^{2} \backslash\{0\}$ and $y \in S$, while $\mathcal{L}_{S}^{(q)}: H^{\frac{1}{2}}(S) \rightarrow H^{-\frac{1}{2}}(S)$ are strongly elliptic pseudodifferential operators of order 1 with strictly negative principal homogenous symbols $-\frac{1}{2} a_{q}(y)\left|\xi^{\prime}\right|$ for $\xi^{\prime} \in \mathbb{R}^{2} \backslash\{0\}$ and $y \in S$. Therefore by standard arguments it can be shown that the operators in the main diagonal in (1.30) are Fredholm of zero index in the corresponding function spaces. Therefore the operator (1.29) is also Fredholm with zero index.

It remains to show that the null space of the operator (1.29) is trivial. We proceed as follows. Let $U \in \mathbb{X}^{(T D)}$ be a solution to the homogeneous system $\mathcal{K}^{(T D)} U=0$. Then the first two equations of the system imply that $U \in \mathbb{H}^{(T D)}$ due Theorems 6 and 7 , and by the equivalence Theorem 1 we conclude $U=0$. Thus the kernel of the operator (1.29) is trivial and consequently (1.29) is invertible.

To prove invertibility of operator (1.28), we remark that for any $\mathcal{F}^{(T D)} \in$ $\mathbb{F}^{(T D)}$ a unique solution $U \in \mathbb{X}^{(T D)}$ of equation (1.27) is delivered by the inverse to the operator (1.29). On the other hand, since $\mathcal{F}^{(T D)} \in \mathbb{F}^{(T D)}$, the first two lines of the matrix operator $\mathcal{K}^{(T D)}$ imply that in fact $U \in \mathbb{H}^{(T D)}$ and the mapping $\mathbb{F}^{(T D)} \rightarrow \mathbb{H}^{(T D)}$ delivered by the inverse to the operator (1.29) is continuous, i.e., this operator is inverse to operator (1.28). 


\subsection{The transmission mixed problem (TM)}

Let us consider the mixed type transmission problem (1.3), (1.4), (1.5), (1.7), (1.8), with the right hand sides

$$
\begin{array}{ll}
\varphi_{0 i} \in H^{\frac{1}{2}}\left(S_{i}\right), & \psi_{0 i} \in H^{-\frac{1}{2}}\left(S_{i}\right), \\
\varphi_{0 e}^{(M)} \in H^{\frac{1}{2}}\left(S_{e D}\right), & \psi_{0 e}^{(M)} \in H^{-\frac{1}{2}}\left(S_{e N}\right), \quad f_{q} \in H^{0}\left(\Omega_{q}\right), \quad q=1,2 .
\end{array}
$$

Let us denote by $\Phi_{0 e} \in H^{\frac{1}{2}}\left(S_{e}\right)$ and $\Psi_{0 e} \in H^{-\frac{1}{2}}\left(S_{e}\right)$ some fixed extensions of the boundary functions $\varphi_{0 e}^{(M)}$ and $\psi_{0 e}^{(M)}$ from $S_{e D}$ and $S_{e N}$, respectively, onto the whole surface $S_{e}$, preserving the space. Then $r_{S_{e D}} \Phi_{0 e}=\varphi_{0 e}^{(M)}, \quad r_{S_{e N}} \Psi_{0 e}=$ $\psi_{0 e}^{(M)}$.

Similar to (1.19) for the Problem (TD), let us introduce the following combinations of the unknown boundary functions

$$
\begin{array}{ll}
\psi_{i}=\frac{1}{2}\left(T_{1} u_{1}-T_{2} u_{2}\right) \in H^{-\frac{1}{2}}\left(S_{i}\right), & \varphi_{i}=\frac{1}{2}\left(\gamma_{1} u_{1}+\gamma_{2} u_{2}\right) \in H^{\frac{1}{2}}\left(S_{i}\right), \\
\psi_{e}=T_{2} u_{2}-\Psi_{0 e} \in \widetilde{H}^{-\frac{1}{2}}\left(S_{e D}\right), & \varphi_{e}=\gamma_{2} u_{2}-\Phi_{0 e} \in \widetilde{H}^{\frac{1}{2}}\left(S_{e N}\right) .
\end{array}
$$

Further, let us set

$$
\begin{aligned}
U^{(T M)}:=\left(u_{1}, u_{2}, \psi_{i}, \varphi_{i}, \psi_{e}, \varphi_{e}\right)^{\top} \in \mathbb{H}^{(T M)} & \\
\mathbb{H}^{(T M)}:=H^{1,0}\left(\Omega_{1} ; A_{1}\right) \times H^{1,0}\left(\Omega_{2} ; A_{2}\right) \times & H^{-\frac{1}{2}}\left(S_{i}\right) \times H^{\frac{1}{2}}\left(S_{i}\right) \\
& \times \widetilde{H}^{-\frac{1}{2}}\left(S_{e D}\right) \times \widetilde{H}^{\frac{1}{2}}\left(S_{e N}\right),
\end{aligned}
$$

and we consider again the components of the vector $U^{(T M)}$ as formally unrelated.

Let us employ the third Green identities (1.15) in $\Omega_{1}$ and $\Omega_{2}$, difference of their traces (1.16) and sum of their co-normal derivatives (1.17) on $S_{i}$, and also the trace (1.16) on $S_{e D}$ and the co-normal derivative (1.17) on $S_{e N}$. Then after substituting transmission conditions (1.4)-(1.5) and mixed boundary conditions (1.7)-(1.8) we arrive at the following system of direct segregated LBDIEs for the components of the vector $U^{(T M)}$,

$$
\begin{aligned}
& u_{1}+\mathcal{R}_{1} u_{1}-V_{S_{i}}^{(1)} \psi_{i}+W_{S_{i}}^{(1)} \varphi_{i}=F_{1}^{(T M)} \text { in } \Omega_{1}, \\
& u_{2}+\mathcal{R}_{2} u_{2}+V_{S_{i}}^{(2)} \psi_{i}+W_{S_{i}}^{(2)} \varphi_{i}-V_{S_{e}}^{(2)} \psi_{e}+W_{S_{e}}^{(2)} \varphi_{e}=F_{2}^{(T M)} \text { in } \Omega_{2}, \\
& \gamma_{1} \mathcal{R}_{1} u_{1}-\gamma_{2} \mathcal{R}_{2} u_{2}-\left(\mathcal{V}_{S_{i}}^{(1)}+\mathcal{V}_{S_{i}}^{(2)}\right) \psi_{i}+\left(\mathcal{W}_{S_{i}}^{(1)}-\mathcal{W}_{S_{i}}^{(2)}\right) \varphi_{i} \\
& \quad \quad+\gamma_{2} V_{S_{e}}^{(2)} \psi_{e}-\gamma_{2} W_{S_{e}}^{(2)} \varphi_{e}=\gamma_{1} F_{1}^{(T M)}-\gamma_{2} F_{2}^{(T M)}-\varphi_{0 i} \text { on } S_{i}, \\
& T_{1} \mathcal{R}_{1} u_{1}+T_{2} \mathcal{R}_{2} u_{2}-\left(\mathcal{W}_{S_{i}}^{(1)}-\mathcal{W}_{S_{i}}^{(2)}\right) \psi_{i}+\left(\mathcal{L}_{S_{i}}^{(1)}+\mathcal{L}_{S_{i}}^{(2)}\right) \varphi_{i} \\
& \quad-T_{2} V_{S_{e}}^{(2)} \psi_{e}+T_{2} W_{S_{e}}^{(2)} \varphi_{e}=T_{1} F_{1}^{(T M)}+T_{2} F_{2}^{(T M)}-\psi_{0 i} \text { on } S_{i},
\end{aligned}
$$




$$
\begin{gathered}
\gamma_{2} \mathcal{R}_{2} u_{2}+\gamma_{2} V_{S_{i}}^{(2)} \psi_{i}+\gamma_{2} W_{S_{i}}^{(2)} \varphi_{i}-\mathcal{V}_{S_{e}}^{(2)} \psi_{e}+\mathcal{W}_{S_{e}}^{(2)} \varphi_{e} \\
=\gamma_{2} F_{2}^{(T M)}-\varphi_{0 e} \text { on } S_{e D}, \\
T_{2} \mathcal{R}_{2} u_{2}+T_{2} V_{S_{i}}^{(2)} \psi_{i}+T_{2} W_{S_{i}}^{(2)} \varphi_{i}-\mathcal{W}_{S_{e}}^{(2)} \psi_{e}+\mathcal{L}_{S_{e}}^{(2)} \varphi_{e} \\
=T_{2} F_{2}^{(T M)}-\psi_{0 e} \text { on } S_{e N},
\end{gathered}
$$

where

$$
\begin{aligned}
& F_{1}^{(T M)}=\mathcal{P}_{1} f_{1}+\frac{1}{2} V_{S_{i}}^{(1)} \psi_{0 i}-\frac{1}{2} W_{S_{i}}^{(1)} \varphi_{0 i} \\
& F_{2}^{(T M)}=\mathcal{P}_{2} f_{2}+\frac{1}{2} V_{S_{i}}^{(2)} \psi_{0 i}+\frac{1}{2} W_{S_{i}}^{(2)} \varphi_{0 i}+V_{S_{e}}^{(2)} \Psi_{0 e}-W_{S_{e}}^{(2)} \Phi_{0 e}
\end{aligned}
$$

As in the case of the problem (TD), we have here the following equivalence theorem.

Theorem 3. Let $\chi \in X_{1 *}^{3}$ and conditions (1.32) hold. Further, let $\Phi_{0 e} \in$ $H^{\frac{1}{2}}\left(S_{e}\right)$ and $\Psi_{0 e} \in H^{-\frac{1}{2}}\left(S_{e}\right)$ be some fixed extensions of the boundary functions $\varphi_{0 e}^{(M)}$ and $\psi_{0 e}^{(M)}$ from $S_{e D}$ and $S_{e N}$, respectively, onto the whole surface $S_{e}$.

(i) If a pair $\left(u_{1}, u_{2}\right) \in H^{1,0}\left(\Omega_{1} ; A_{1}\right) \times H^{1,0}\left(\Omega_{2} ; A_{2}\right)$ solves the transmission mixed problem (TM), then the six-vector $U^{(T M)} \in \mathbb{H}^{(T M)}$ given by (1.34), where $\psi_{i}, \varphi_{i}, \psi_{e}$ and $\varphi_{e}$ are defined by (1.33), solves the LBDIEs system (1.35)-(1.42).

(ii) Vice versa, if a six-vector $U^{(T M)} \in \mathbb{H}^{(T M)}$ solves the LBDIEs system (1.35)-(1.42) and condition (1.14) holds, then the pair $\left(u_{1}, u_{2}\right)$ solves the Problem (TM) and the relations (1.33) hold.

Proof. The claim (i) immediately follows from the deduction of (1.35)-(1.42).

Now, let a six-vector $U^{(T M)}$ solve the LBDIEs system (1.35)-(1.42). Subtracting from equation (1.37) the trace $\gamma_{1}$ of equation (1.35) and adding the trace $\gamma_{2}$ of equation (1.36), we prove (1.4). Similarly, subtracting from equation (1.38) the co-normal derivative $T_{1}$ of equation (1.35) and the co-normal derivative $T_{2}$ of equation (1.36), we prove (1.5). Subtracting from equation (1.39) the trace $\gamma_{2}$ of equation (1.36), we prove (1.7). Similarly, subtracting from equation (1.40) the co-normal derivative $T_{2}$ of equation (1.36), we prove (1.8). That is, the transmission conditions on $S_{i}$ and the mixed boundary conditions on $S_{e}$ are fulfilled.

It remains to show that the equations (1.3) and the relations (1.33) hold true. Due to the embedding $U^{(T M)} \in \mathbb{H}^{(T M)}$, the third Green identities (1.15) hold. Comparing these identities with the first two equations of the LBDIEs system, (1.35) and (1.36), and taking into account transmission conditions (1.4)-(1.5) and mixed boundary conditions (1.7)-(1.8), already satisfied, we arrive at the relations 


$$
\begin{aligned}
& V_{S_{i}}^{(1)}\left(\frac{1}{2}\left[T_{1} u_{1}-T_{2} u_{2}\right]-\psi_{i}\right)+W_{S_{i}}^{(1)}\left(\varphi_{i}-\frac{1}{2}\left[\gamma_{1} u_{1}+\gamma_{2} u_{2}\right]\right) \\
& \quad=\mathcal{P}_{1}\left(f_{1}-A_{1} u_{1}\right) \text { in } \Omega_{1}, \\
& \begin{array}{r}
V_{S_{i}}^{(2)}\left(\frac{1}{2}\left[T_{1} u_{1}-T_{2} u_{2}\right]-\psi_{i}\right)-W_{S_{i}}^{(2)}\left(\varphi_{i}-\frac{1}{2}\left[\gamma_{1} u_{1}+\gamma_{2} u_{2}\right]\right) \\
+V_{S_{e}}^{(2)}\left(-T_{2} u_{2}+\psi_{e}+\Psi_{0 e}\right)+W_{S_{e}}^{(2)}\left(\gamma_{2} u_{2}-\varphi_{e}-\Phi_{0 e}\right) \\
=\mathcal{P}_{2}\left(A_{2} u_{2}-f_{2}\right) \text { in } \Omega_{2} .
\end{array}
\end{aligned}
$$

Whence by Lemma 2 we conclude that (1.3) and (1.33) are satisfied.

Denote by $\mathcal{K}^{(T M)}=\left[\mathcal{K}_{k j}^{(T M)}\right]_{6 \times 6}$ the localized boundary-domain $6 \times 6$ matrix integral operator generated by the left hand side expression in (1.35)(1.40) and set

$$
\begin{aligned}
\mathbb{F}^{(T M)}:=H^{1,0}\left(\Omega_{1} ; A_{1}\right) \times H^{1,0}\left(\Omega_{2} ; A_{2}\right) & \times H^{\frac{1}{2}}\left(S_{i}\right) \times H^{-\frac{1}{2}}\left(S_{i}\right) \\
& \times H^{\frac{1}{2}}\left(S_{e D}\right) \times H^{-\frac{1}{2}}\left(S_{e N}\right) .
\end{aligned}
$$

Then the LBDIEs system (1.35)-(1.40) is written in matrix form as

$$
\mathcal{K}^{(T M)} U^{(T M)}=\mathcal{F}^{(T M)},
$$

where $U^{(T M)}$ is the unknown six-vector function (1.34), while $\mathcal{F}^{(T M)} \in \mathbb{F}^{(T M)}$ is the known vector function compiled by the right hand side functions in (1.35)-(1.40).

From Theorem 3 it follows that the LBDIEs system (1.35)-(1.40), i.e., equation (1.44), is uniquely solvable in the space $\mathbb{H}^{(T M)}$ for the special right hand side vector-function, which belongs to the space $\mathbb{F}^{(T M)}$ defined by (1.43). One can easily check that the right hand side expressions in LBDIEs system (1.35)-(1.40) vanish if $f_{q}=0$ in $\Omega_{q}, q=1,2, f_{1}=0$ and $\psi_{0 i}=0$ on $S_{i}$, $\Phi_{0 e}=0$ and $\Psi_{0 e}=0$ on $S_{e}$.

Now we establish that actually the operator given by the left hand side of equation (1.44) is continuously invertible as an operator both in the function spaces already introduced and in wider function spaces. To this end let us consider the operators

$$
\begin{aligned}
\mathcal{K}^{(T M)} & : \mathbb{H}^{(T M)} \rightarrow \mathbb{F}^{(T M)}, \\
& : \mathbb{X}^{(T M)} \rightarrow \mathbb{Y}^{(T M)},
\end{aligned}
$$

where

$$
\begin{aligned}
& \mathbb{X}^{(T M)}:=H^{1}\left(\Omega_{1}\right) \times H^{1}\left(\Omega_{2}\right) \times H^{-\frac{1}{2}}\left(S_{i}\right) \times H^{\frac{1}{2}}\left(S_{i}\right) \times \widetilde{H}^{-\frac{1}{2}}\left(S_{e D}\right) \times \widetilde{H}^{\frac{1}{2}}\left(S_{e N}\right), \\
& \mathbb{Y}^{(T M)}:=H^{1}\left(\Omega_{1}\right) \times H^{1}\left(\Omega_{2}\right) \times H^{\frac{1}{2}}\left(S_{i}\right) \times H^{-\frac{1}{2}}\left(S_{i}\right) \times H^{\frac{1}{2}}\left(S_{e D}\right) \times H^{-\frac{1}{2}}\left(S_{e N}\right) .
\end{aligned}
$$

As follows from the mapping properties of the potentials (see Theorems 6 and 7), operators (1.45) and (1.46) are bounded. 
Let us show that operator (1.46) is Fredholm with zero index and thus (1.46) and consequently (1.45) are invertible.

Consider the upper triangular operator

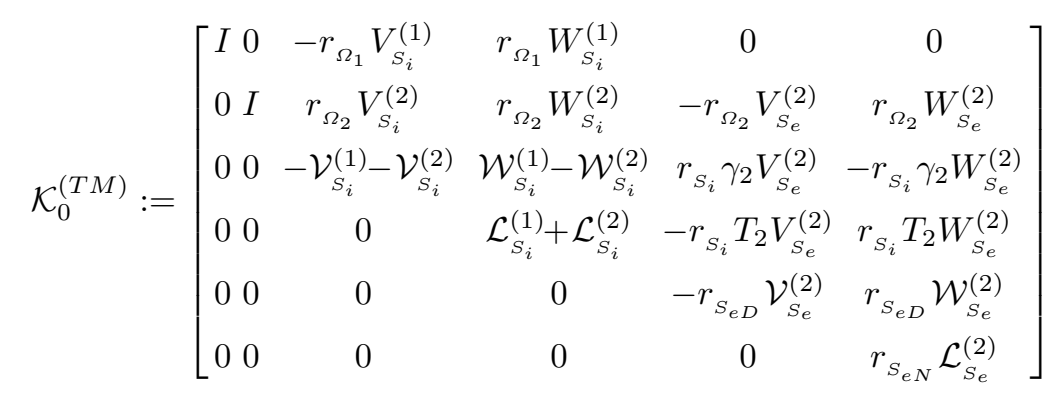

It is easy to see that, on the one hand, the operator

$$
\mathcal{K}_{0}^{(T M)}: \mathbb{X}^{(T M)} \rightarrow \mathbb{Y}^{(T M)}
$$

is bounded, while due to Lemma 4,

$$
\mathcal{K}^{(T M)}-\mathcal{K}_{0}^{(T M)}: \mathbb{X}^{(T M)} \rightarrow \mathbb{Y}^{(T M)}
$$

is a compact operator.

On the other hand, as it has been mentioned above, in the proof of Theorem 2 , the third and forth operators in the main diagonal

$$
\begin{aligned}
& -\left[\mathcal{V}_{S_{i}}^{(1)}+\mathcal{V}_{S_{i}}^{(2)}\right]: H^{-\frac{1}{2}}\left(S_{i}\right) \rightarrow H^{\frac{1}{2}}\left(S_{i}\right), \\
& \mathcal{L}_{S_{i}}^{(1)}+\mathcal{L}_{S_{i}}^{(2)}: H^{\frac{1}{2}}\left(S_{i}\right) \rightarrow H^{-\frac{1}{2}}\left(S_{i}\right),
\end{aligned}
$$

are Fredholm with zero index.

Moreover, applying the results of the theory of strongly elliptic pseudodifferential equations on manifolds with boundary (see, e.g., [BCN09, Theorem 3.5], [CMN09-1, Lemma 3.4]), we conclude that the last two operators on the main diagonal,

$$
\begin{aligned}
& r_{S_{e D}} \mathcal{V}_{S_{e}}^{(2)}: \widetilde{H}^{-\frac{1}{2}}\left(S_{e D}\right) \rightarrow H^{\frac{1}{2}}\left(S_{e D}\right), \\
& r_{S_{e N}} \mathcal{L}_{S_{e}}^{(2)}: \widetilde{H}^{\frac{1}{2}}\left(S_{e N}\right) \rightarrow H^{-\frac{1}{2}}\left(S_{e N}\right),
\end{aligned}
$$

are also Fredholm operators with zero index.

Therefore, (1.47) and consequently (1.46) is a Fredholm operator with zero index. It remains to show that the null space of operator (1.46) is trivial. Let $U \in \mathbb{X}^{(T M)}$ be a solution to the homogeneous equation $\mathcal{K}^{(T M)} U=0$. Then due to the first two lines of the matrix equation and mapping properties (1.52), (1.53) and (1.54) we see that $U \in \mathbb{H}^{(T M)}$ and by the equivalence Theorem 3 we conclude $U=0$ due to the uniqueness theorem for the problem (TM) in the space $\mathbb{H}^{(T M)}$. Thus the operator (1.46) is invertible. 
To prove the invertibility of operator (1.45), we note that for any $\mathcal{F}^{(T M)} \in$ $\mathbb{F}^{(T M)}$ a unique solution $U \in \mathbb{X}^{(T M)}$ of equation (1.44) is delivered by the inverse to the operator (1.46). On the other hand, since $\mathcal{F}^{(T M)} \in \mathbb{F}^{(T M)}$, the first two lines of the matrix operator $\mathcal{K}^{(T M)}$ imply that in fact $U \in \mathbb{H}^{(T M)}$ and the mapping $\mathbb{F}^{(T M)} \rightarrow \mathbb{H}^{(T M)}$ delivered by the inverse to the operator (1.46) is continuous, i.e., this operator gives inverse to operator (1.45) as well.

Now we can summarize the results obtained above in the following theorem.

Theorem 4. Let $\chi \in X_{1 *}^{3}$ and condition (1.14) hold. Then the operators (1.45) and (1.46) are invertible.

\subsection{Appendix A: Classes of localizing functions}

Let us introduce the classes for localizing functions (cf. [CMN09-L]).

Definition 1. (i) We say $\chi \in X^{k}$ for integer $k \geq 0$ if $\chi(x)=\breve{\chi}(|x|), \quad \breve{\chi} \in$ $W_{1}^{k}(0, \infty), \quad \varrho \breve{\chi}(\varrho) \in L_{1}(0, \infty)$.

(ii) We say $\chi \in X_{*}^{k}$ for $k \geq 1$ if $\chi \in X^{k}, \chi(0)=1$ and

$$
\sigma_{\chi}(\omega):=\frac{1}{\omega} \widehat{\chi}_{s}(\omega)>0 \text { for a.e. } \omega \in \mathbb{R},
$$

where $\widehat{\chi}_{s}(\omega)$ denotes the sine-transform of $\breve{\chi}: \widehat{\chi}_{s}(\omega):=\int_{0}^{\infty} \breve{\chi}(\varrho) \sin (\varrho \omega) d \varrho$.

(iii) We say $\chi \in X_{1 *}^{k}$ for $k \geq 1$ if $\chi \in X_{*}^{k}$ and $\omega \widehat{\chi}_{s}(\omega) \leq 1 \quad \forall \omega \in \mathbb{R}$.

Note that if $\breve{\chi} \in W^{k}(0, \infty), k \geq 1$, then $\breve{\chi}$ is continuous due to the Sobolev embedding theorem, and $\chi(0)=\breve{\chi}(0)$ is well defined by continuity of $\breve{\chi}$. Evidently, we have the following imbeddings: $X^{k_{1}} \subset X^{k_{2}}, X_{*}^{k_{1}} \subset X_{*}^{k_{2}}$, and $X_{1 *}^{k_{1}} \subset X_{1 *}^{k_{2}}$ for $k_{1}>k_{2}$. Since the inequality in (1.48) is to be satisfied only almost everywhere, the classes $X_{*}^{k}, X_{1 *}^{k}$ are wider than their corresponding counterparts $X_{+}^{k}, X_{1+}^{k}$ from [CMN09-L].

Some examples of the functions $\chi$ from these classes are presented in [CMN09-L].

The class $X_{*}^{k}$ is defined in terms of the sine-transform. The following lemma implied by [CMN09-L, Lemma 3.2] gives an easily verifiable sufficient condition for non-negative non-increasing functions to belong to the class $X_{*}^{k} \supset X_{+}^{k}$.

Lemma 3. If $\chi \in X^{k}, k \geq 1, \breve{\chi}(0)=1, \breve{\chi}(\varrho) \geq 0$ for all $\varrho \in(0, \infty)$, and $\breve{\chi}$ is a non-increasing function on $[0,+\infty)$, then $\chi \in X_{*}^{k}$.

\subsection{Appendix B: Properties of localized potentials}

Here we collect some assertions describing the properties of the localized potentials following from [CMN09-1, CMN09-L]. 
Theorem 5. The following operators are continuous

$$
\begin{aligned}
& \mathcal{P}_{q}: \widetilde{H}^{s}\left(\Omega_{q}\right) \rightarrow H^{s+2}\left(\Omega_{q}\right), \quad s \in \mathbb{R}, \quad \chi \in X^{1}, \\
& \mathcal{P}_{q}: H^{s}\left(\Omega_{q}\right) \rightarrow H^{s+2}\left(\Omega_{q}\right), \quad-\frac{1}{2}<s<k-\frac{1}{2}, \quad \chi \in X^{k}, k=1,2,3 .
\end{aligned}
$$

Continuity of (1.49) is given by [CMN09-L, Theorem 5.4] while (1.50) can be proved using [CMN09-L, Lemma 5.9] and [CMN09-1, Theorem 3.8].

Theorem 6. The following operators are continuous

$$
\begin{aligned}
\mathcal{R}_{q}: \widetilde{H}^{s}\left(\Omega_{q}\right) & \rightarrow H^{s+1}\left(\Omega_{q}\right), \quad s \in \mathbb{R}, \quad \chi \in X^{2}, \\
\mathcal{R}_{q}: H^{s}\left(\Omega_{q}\right) & \rightarrow H^{t}\left(\Omega_{q}\right), \quad-\frac{1}{2}<s<k-\frac{1}{2}, \\
& t<k-\frac{1}{2}, \quad t \leq s+1, \quad \chi \in X^{k}, \quad k=2,3 .
\end{aligned}
$$

Continuity of (1.51) is given by [CMN09-L, Theorem 5.4] while (1.52) can be proved using the continuity of operator (1.50) above along with relation (3.28) and Lemma 5.3 from [CMN09-L].

Theorem 6 implies the following statement.

Lemma 4. The operators

$$
\begin{aligned}
& \mathcal{R}_{q}: H^{1}\left(\Omega_{q}\right) \rightarrow H^{t}\left(\Omega_{q}\right), \quad t<3 / 2, \quad \chi \in X^{2}, \\
& \gamma_{q} \mathcal{R}_{q}: H^{1}\left(\Omega_{q}\right) \rightarrow H^{t-\frac{1}{2}}\left(\partial \Omega_{q}\right), \quad t<3 / 2, \quad \chi \in X^{2}, \\
& T_{q} \mathcal{R}_{q}: H^{1}\left(\Omega_{q}\right) \rightarrow H^{t-\frac{3}{2}}\left(\partial \Omega_{q}\right), \quad t<2, \quad \chi \in X^{3}
\end{aligned}
$$

are compact.

Theorem 7. The following localized operators are continuous

$$
\begin{aligned}
& V_{S}^{(q)}: H^{-\frac{1}{2}}(S) \rightarrow H^{1,0}\left(\Omega_{q}^{ \pm} ; A_{q}\right), \quad \chi \in X^{2}, \\
& W_{S}^{(q)}: H^{\frac{1}{2}}(S) \rightarrow H^{1,0}\left(\Omega_{q}^{ \pm} ; A_{q}\right), \quad \chi \in X^{3}, \\
& \mathcal{V}_{S}^{(q)}: H^{-\frac{1}{2}}(S) \rightarrow H^{\frac{1}{2}}(S), \quad \chi \in X^{1}, \\
& \mathcal{W}_{S}^{\prime(q)}: H^{-\frac{1}{2}}(S) \rightarrow H^{-\frac{1}{2}}(S), \quad \chi \in X^{2}, \\
& \mathcal{W}_{S}^{(q)}: H^{\frac{1}{2}}(S) \rightarrow H^{\frac{1}{2}}(S), \quad \chi \in X^{2}, \\
& \mathcal{L}_{S}^{ \pm(q)}: H^{\frac{1}{2}}(S) \rightarrow H^{-\frac{1}{2}}(S) \quad \chi \in X^{3},
\end{aligned}
$$

where $\Omega_{q}^{+}:=\Omega_{q}, \Omega_{q}^{-}:=\mathbb{R}^{3} \backslash \bar{\Omega}_{q}$.

Theorem 7 follows from [CMN09-L, Theorems 5.10, 5.14].

The following jump properties are given by [CMN09-L, Theorem 5.13]. 
Theorem 8. Let $g \in H^{-\frac{1}{2}}(S)$ and $h \in H^{\frac{1}{2}}(S)$. Then

$$
\begin{aligned}
& \gamma_{q}^{+} V_{S}^{(q)} g=\gamma_{q}^{-} V_{S}^{(q)} g=\mathcal{V}_{S}^{(q)} g, \quad \chi \in X^{1}, \\
& T_{q}^{ \pm} V_{S}^{(q)} g= \pm \frac{1}{2} g+\mathcal{W}_{S}^{\prime(q)} g, \quad \chi \in X^{2}, \\
& \gamma_{q}^{ \pm} W_{S}^{(q)} h=\mp \frac{1}{2} h+\mathcal{W}_{S}^{(q)} h, \quad \chi \in X^{2}, \\
& T_{q}^{+} W_{S}^{(q)} h-T_{q}^{-} W_{S}^{(q)} h \equiv \mathcal{L}_{S}^{+(q)} h-\mathcal{L}_{S}^{-(q)} h=h \frac{\partial a_{q}}{\partial n^{(q)}}, \chi \in X^{3} .
\end{aligned}
$$

Acknowledgements. This research was supported by the EPSRC grant EP/H020497/1: "Mathematical analysis of Localized Boundary-Domain Integral Equations for Variable-Coefficient Boundary Value Problems" and partly by the Georgian Technical University grant in the case of the third author.

\section{References}

[BCN09] Buchukuri, T., Chkadua, O., Natroshvili, D.: Mixed boundary value problems of thermopiezoelectricity for solids with interior cracks, Integral Equations and Operator Theory, 64, 4, 495-537 (2009).

[CMN09-1] Chkadua, O., Mikhailov, S., Natroshvili, D.: Analysis of direct boundary-domain integral equations for a mixed BVP with variable coefficient. Part I. Equivalence and invertibility. J. Integral Equations Appl. 21, 499-542. (2009).

[CMN09-L] Chkadua, O., Mikhailov, S., Natroshvili, D.: Analysis of some localized boundary-domain integral equations. J. Integral Equations Appl. 21, 407-447 (2009).

[CMN11] Chkadua, O., Mikhailov, S. E., Natroshvili, D.: Analysis of segregated boundary-domain integral equations for variable-coefficient problems with cracks. Numerical Methods for PDEs, 27(1), 121-140 (2011).

[Co88] Costabel, M.: Boundary integral operators on Lipschitz domains: elementary results. SIAM J. Math. Anal., 19, 613-626 (1988).

[DaLi90] Dautray, R., Lions, J.L.: Mathematical analysis and numerical methods for science and technology, Vol. 4, Integral equations and numerical methods, Springer-Verlag, Berlin (1990).

[HW08] Hsiao, G.C., Wendland, W.L.: Boundary Integral Equations, Applied Mathematical Sciences, Springer-Verlag, Berlin-Heidelberg (2008).

[LiMa72] Lions, J.-L., Magenes, E.: Non-homogeneous boundary value problems and applications, Vol. 1, Springer-Verlag, New York-Heidelberg (1972).

[McL00] McLean, W.: Strongly Elliptic Systems and Boundary Integral Equations. Cambridge University Press, Cambridge, UK (2000).

[Mik02] Mikhailov, S.E.: Localized boundary-domain integral formulation for problems with variable coefficients, Int. J. Engineering Analysis with Boundary Elements 26, 681-690 (2002). 
[Mik06] Mikhailov, S. E.: Analysis of united boundary-domain integrodifferential and integral equations for a mixed BVP with variable coefficient. Math. Methods in Applied Sciences, 29, 715-739 (2006).

[MN05] Mikhailov, S.E., Nakhova, I.S.: Mesh-based numerical implementation of the localized boundary-domain integral equation method to a variable-coefficient Neumann problem. J. Engineering Math. 51, 251259 (2005).

[Mir70] Miranda, C.: Partial differential equations of elliptic type, Second revised edition, Springer-Verlag, New York-Berlin (1970).

[SSA00] Sladek, J., Sladek, V., Atluri, S.N.: Local boundary integral equation (LBIE) method for solving problems of elasticity with nonhomogeneous material properties, Comput. Mech., 24, 6, 456-462 (2000).

[ZZA98] Zhu, T., Zhang, J.-D., Atluri, S.N.: A local boundary integral equation (LBIE) method in computational mechanics, and a meshless discretization approach, Comput. Mech., 21, 3, 223-235 (1998).

[ZZA99] Zhu, T., Zhang, J.-D., Atluri, S.N.: A meshless numerical method based on the local boundary integral equation (LBIE) to solve linear and nonlinear boundary value problems, Eng. Anal. Bound. Elem., 23, 5-6, 375-389 (1999). 